\title{
The Mystery of Nature's Toughest Material
}

\begin{abstract}
Nacre, the rainbow-sheened material that lines the inside of mollusk shells, has mystified scientists for more than 80 years. Long hailed among the toughest material on earth, its combination of hardness and resilience is stronger than anything yet created by humans. Now, a team of scientistst the University of Erlangen, the University of Michigan and elsewhere have revealed, for the first time, precisely how the mollusks are so tough. With the results, a new generation of break-resistant ceramic materials can be developed.
\end{abstract}

Researchers have known the basics about nacre for decades-it's made of microscopic "bricks" of a mineral called aragonite, laced together with a "mortar" made of organic material. This brick-and-mortar arrangement clearly lends strength, but nacre is far more resilient than its materials suggest. An interdisciplinary team around the globe worked together to crack the mystery. Using tiny piezo-electric micro-indenters, they were able to exert force on the shells while

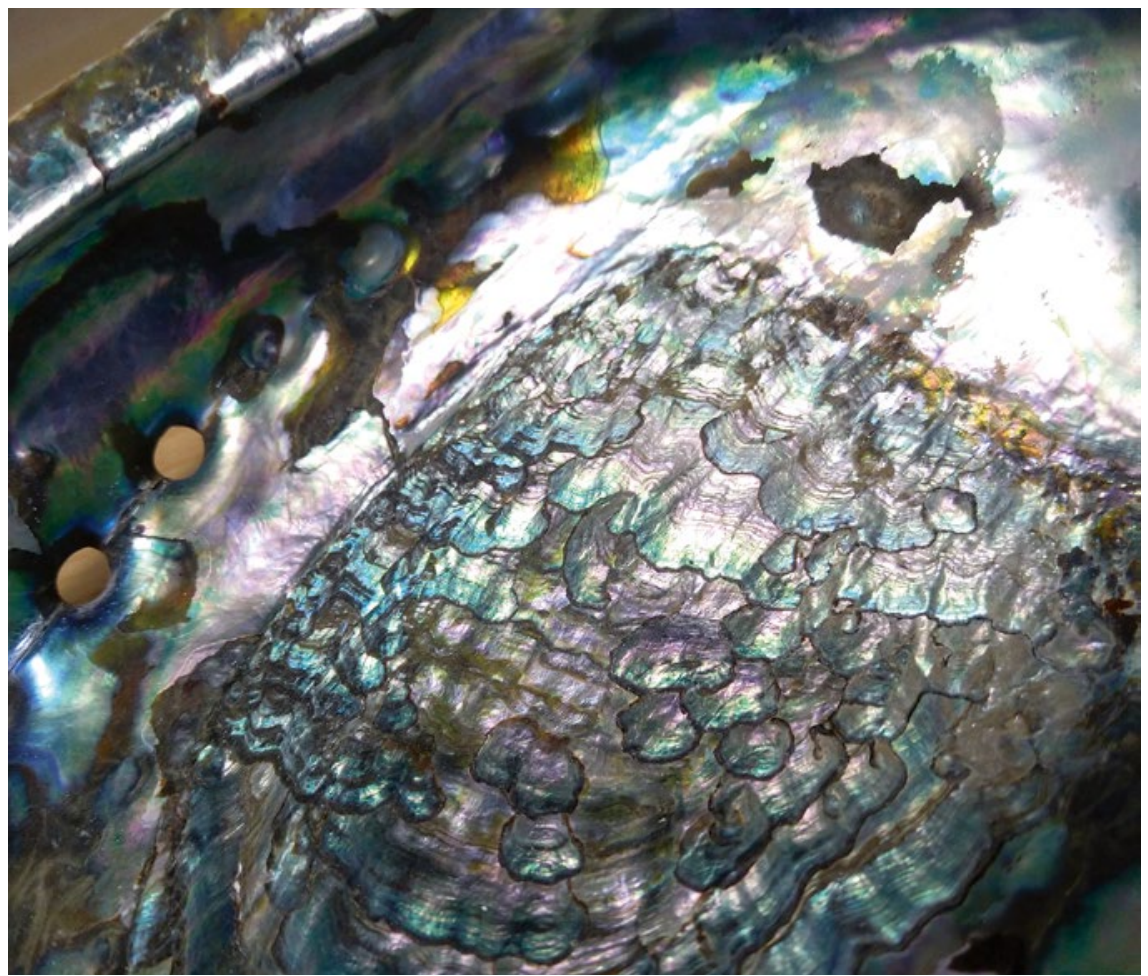

Figure 1 Together with international colleagues, FAU scientists have cracked the mystery of the hardness of mother-of-pearl (the picture shows the mother-of-pearl of an abalone snail). (৫ Stephan E. Wolf) they were under an electron microscope and watch what happened in real time. They revealed that the structure deforms with more complexity than imagined.

"The grain structure on the nanoscale is key to this hierarchical and hybrid organization. It arises from tiny particles which assemble on the nanoscale. In order to use our findings for future applications, we have to understand these exceptional processes in detail since they eventually lead to a ceramic mate- rial with fantastic properties," says Stephan Wolf. He is Juniorprofessor for Biomimetic Materials and Processes at Friedrich-Alexander-University Erlangen-Nuremberg.

They found that the "bricks" are actually multi-sided tablets only a few hundred nanometers in size. Ordinarily, these tablets remain separate, arranged in layers and cushioned by a thin layer of organic "mortar." But when stress is applied to the shells, the "mortar" squishes aside and the tablets lock together, forming what is essentially a solid surface. When the force is removed, the structure springs back, without losing any strength or resilience. This resilience sets nacre apart from even the most advanced human-designed materials. Plastics, for example, can spring back from an impact, but they lose some of their strength each time. Nacre lost none of its resilience in repeated impacts at up to $80 \%$ of its yield strength. What's more, if a crack does form, nacre confines the crack to a single layer rather than allowing it to spread, keeping the shell's structure intact-the mollusk lives another day. In essence, the material gains its strength by combining the advantages of solid and layered structures.

"It's incredible that a mollusk, which is not the most intelligent creature, is fabricating such complex structures across so many scales," Robert Hovden from the University of Michigan and head of the study, said.

Contact:

University of Erlangen-Nuremberg, Institute of Glass and Ceramics, Erlangen,Germany www.ww.tf.fau.de 